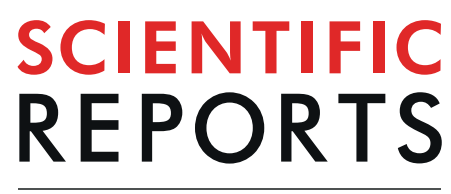

natureresearch

Check for updates

\title{
Importance of Thyroid Hormone level and Genetic Variations in Deiodinases for Patients after Acute Myocardial Infarction: A Longitudinal Observational Study
}

\author{
Nijole Kazukauskiene ${ }^{1}$, Daina Skiriute ${ }^{2}$, Olivija Gustiene ${ }^{3}$, Julius Burkauskas ${ }^{4 凶}$, \\ Violeta Zaliunaite ${ }^{5}$, Narseta Mickuviene ${ }^{6}$ \& Julija Brozaitiene ${ }^{4}$
}

This study aimed to examine the influence of thyroid hormone (TH) levels and genetic polymorphisms of deiodinases on long-term outcomes after acute myocardial infarction (AMI). In total, 290 patients who have experienced AMI were evaluated for demographic, clinical characteristics, risk factors, TH and NT-pro-BNP. Polymorphisms of TH related genes were included deiodinase 1 (DIO1) (rs11206244-C/T, rs12095080-A/G, rs2235544-A/C), deiodinase 2 (DIO2) (rs225015-G/A, rs225014-T/C) and deiodinase 3 (DIO3) (rs945006-T/G). Both all-cause and cardiac mortality was considered key outcomes. Cox regression model showed that NT-pro-BNP $(\mathrm{HR}=2.11 ; 95 \% \mathrm{Cl}=1.18-3.78 ; \mathrm{p}=0.012)$, the first quartile of $\mathrm{fT} 3$, and $D I O 1$ gene rs 12095080 were independent predictors of cardiac-related mortality $(\mathrm{HR}=1.74 ; 95 \% \mathrm{Cl}=1.04-2.91 ; \mathrm{p}=0.034)$. The $\mathrm{DIO1}$ gene rs $12095080 \mathrm{AG}$ genotype $(\mathrm{OR}=3.97 ; 95 \%$ $\mathrm{Cl}=1.45-10.89 ; \mathrm{p}=0.005$ ) increased the risk for cardiac mortality. Lower fT3 levels and the DIO1 gene rs12095080 are both associated with cardiac-related mortality after AMI.

Recent clinical research in cardiovascular disease as well as in coronary artery disease (CAD) has provided evidence that altered thyroid hormone (TH) metabolism, including low total triiodothyronine (T3) syndrome or pre-existing subclinical primary hypothyroidism, is an important indicator of adverse short-term and long-term outcomes, including mortality ${ }^{1-5}$. These changes in thyroid homeostasis are known as "euthyroid sick syndrome" ${ }^{6,7}$ or "non-thyroidal illness syndrome" (NTIS) ",9 $^{8}$ and are defined by low serum levels of free T3 (fT3), T3 and high levels of reverse T3 (rT3) followed by normal or low levels of thyroxine (T4) and thyroid-stimulating hormone (TSH). Low T3 syndrome is observed in about one third of patients following acute cardiovascular events and has been linked to the severity of the disease and its adverse prognosis ${ }^{10}$. This syndrome has been established in patients with heart failure $(\mathrm{HF})^{11-14}$, myocardial infarction $(\mathrm{MI})^{2,15-18}$, and has been linked to the cardiac remodelling process ${ }^{19-21}$ and poor prognosis ${ }^{1,3,4,13,22,23}$. Studies suggest that variations of TH within clinically normal ranges, such as isolated reduction in fT3 level or higher level of free T4 (fT4), could constitute a model of abnormal TH metabolism. These variations could act as a risk factor for CAD, in a similar fashion to overt or subclinical hypothyroidism, thereby influencing the occurrence as well as severity of coronary atherosclerosis and its related outcomes ${ }^{2,24-32}$.

\footnotetext{
${ }^{1}$ Laboratory of Behavioral Medicine, Neuroscience Institute, Lithuanian University of Health Sciences, Vyduno al. 4, LT-00135, Palanga, Lithuania. ' ${ }^{2}$ aboratory of Molecular Neurooncology, Neuroscience Institute, Lithuanian University of Health Sciences, Eiveniu str. 4, LT-50161, Kaunas, Lithuania. ${ }^{3}$ Department of Cardiology, Medical Academy, Lithuanian University of Health Sciences, Eiveniu str. 2, LT-50009, Kaunas, Lithuania. ${ }^{4}$ Laboratory of Behavioral Medicine, Neuroscience Institute, Lithuanian University of Health Science, Vyduno al. 4, LT-00135, Palanga, Lithuania. ${ }^{5}$ Laboratory of Behavioral Medicine, Neuroscience Institute, Lithuanian University of Health Science, Lithuanian University of Health Sciences, Eiveniu str. 2, LT-50009, Kaunas, Lithuania. ${ }^{6}$ Laboratory of Behavioral Medicine, Neuroscience Institute, Lithuanian University of Health Science, Lithuanian University of Health Sciences, Vyduno al. 4, LT-00135, Palanga, Lithuania. ${ }^{\bowtie}$ e-mail: julius.burkauskas@Ismuni.It
} 
Recent studies acknowledge the influence of common genetic variations in TH pathway genes on thyroid function ${ }^{33-36}$. The production of $\mathrm{TH}$, in particular the prohormone $\mathrm{T} 4$, is controlled by the hypothalamic-pituitary-thyroid axis, whereas its biological activity is primarily regulated by iodothyronine deiodinases enzymes. Maintenance of euthyroidism at a serum level and peripherally is determined by deiodinase type 1 (DIO1), deiodinase type 2 (DIO2) and deiodinase type 3 (DIO3) ${ }^{37-41}$. Both DIO1 and DIO2 are predominantly activating enzymes and convert T4 to T3 and rT3 to diiodothyronine (T2), while DIO3 inactivates TH and converts $\mathrm{T} 3$ to $\mathrm{T} 2$ and $\mathrm{T} 4$ to $\mathrm{rT} 3^{20,37,38,42,43}$. During recent years, it has been demonstrated that certain genetic polymorphisms in gene coding for deiodinases could alter gene function and are associated with variations in TH levels, such as fT3, fT4, T4 and rT3 in hypothyroid patients, healthy individuals $s^{34,42,44,45}$ and CAD patients ${ }^{46}$.

To our knowledge, there are no reports studying the association between circulating TH ranges and genetic variability of genes related to TH axis on the long-term mortality in CAD patients after acute MI (AMI). Our study aimed to examine the prognostic importance of TH level and genetic polymorphisms DIO1, DIO2, and $\mathrm{DIO} 3$ on long-term outcomes in patients with CAD after AMI.

\section{Methods}

Study population. In total, $330 \mathrm{AMI}$ patients with ST-segment elevation and non ST-segment elevation in the cardiac Intensive Care Unit (ICU) at the Lithuanian University of Health Sciences Hospital were invited to participate in the study. Standard treatment had been given according to the existing guidelines for AMI management ${ }^{47-50}$. Inclusion criteria covered ages over 18 years and an AMI diagnosis. Patients were excluded if they were taking thyroid medications or amiodarone, had increased levels of TSH ( $>4.8 \mathrm{mIU} / \mathrm{l})$, indicating hypothyroidism, reduced TSH $(<0.5 \mathrm{mIU} / \mathrm{l})$, indicating hyperthyroidism, or if they had serious systemic disease (e.g. cancer, autoimmune disease, or chronic renal disease). All eligible participants provided written informed consent. The final study population was comprised of 290 patients with AMI ( $72 \%$ men and $28 \%$ women; mean age, $62 \pm 11$ years).

Study design. Eligible participants were evaluated for socio-demographic factors and clinical characteristics such as history and type of AMI, HF, left ventricular ejection fraction (LVEF), Killip class, and current medication use. Participants were also evaluated for known CAD risk factors, including diabetes mellitus (DM), arterial hypertension $(\mathrm{AH})$, and body mass index $(\mathrm{BMI})$. All patients underwent coronary angiography. The majority of patients were after primary percutaneous coronary intervention (PCI). Troponin I, lipid profiles, N-terminal pro-B-type natriuretic peptide (NT-pro-BNP), TH concentrations, and DIO1, DIO2, DIO3 genetic polymorphisms were evaluated from a blood samples drawn before intervention procedures.

Follow-up data on mortality (time and cause of death) was used in the analysis as a primary outcome of interest. During a period of two-year follow-up, outcome data from 283 of the 290 participants was collected. The data was obtained from death certificates, post-mortem reports, and medical records. When data could not be obtained from these sources, the study team attempted to conduct telephone interviews with participant family members to obtain self-report mortality data or contacted the Causes of Death Register at the Institute of Hygiene of the Lithuanian Ministry of Health. Cardiac and all-cause mortality were ascertained. Documentation of death due to cardiac arrest or arrhythmias, death due to MI or progressive HF were regarded as cardiac-related mortality. The prospective study protocol was approved by The Regional Biomedical Research Ethics Committee and is described elsewhere ${ }^{51}$.

Evaluation of TH and NT-pro-BNP. Blood samples were taken within 24 hours of patients' admission to the ICU. The blood was centrifuged and the serum was frozen at $-80^{\circ} \mathrm{C}$. Serum samples were analysed in a single batch after completion of this study. Serum levels of T3, fT3, fT4, rT3 and TSH were analysed using an automated enzyme immunoassay analyser (Advia Centaur XP; Siemens Osakeyhtio). The normal range for total T3 was $0.89-2.44 \mathrm{nmol} / \mathrm{L}$, fT3 3.50-6.5 pmol/L, fT4 11.50-22.70 pmol/L, rT3 24.50-269.30 pg/mL and TSH 0.55-4.78 $\mathrm{mIU} / \mathrm{L}$. The serum NT-pro-BNP levels were assessed using two-side chemiluminescent immunometric assay with Immulite 2000 immunoassay System; Siemens, Germany. All subjects included in the study were also evaluated for troponin I, lipid concentrations, serum glucose levels and underwent a common blood test.

Genotyping. Six SNPs were evaluated for thyroid axes related genes including DIO1 (rs11206244-C/T, rs12095080-A/G, rs2235544-A/C); DIO2 (rs225014-T/C, rs225015-G/A) DIO3 (rs945006-T/G). SNPs were selected if they were associated with serum TH levels in individual gene studies or based on data from Genome wide association studies ${ }^{45,52,53}$. We used minor allele frequency (MAF) of at least $10 \%$. SNPs sequence in the studied genes - in DIO1 gene locus rs11206244 (c.*29 C > T), rs12095080 (c.*1058 A > G), rs2235544 (c.68234 C > A), DIO2 gene locus rs225014 (p.Thr92Ala), rs225015 (c.*1453 C > T), DIO3 gene locus rs 945006 (c.*529 T > G). Information for genotyped SNPs is represented in Table 1. Genomic DNA was extracted from peripheral blood samples by the salting out procedure as described elsewhere ${ }^{54}$. The genotyping was completed using TaqMan SNP genotyping assays. (Applied Biosystems, Foster City, CA, USA): C_15952583_10 (rs2235544), C_31601225_10(rs12095080), C_334342_20(rs11206244), C_568127_10(rs225015), C_15819951_10 (rs225014), C_7565113_10 (rs945006), and ABI 7900HT real-time PCR Thermocycler (Applied Biosystems, Foster City, CA, USA). Samples were measured in duplicates and nuclease-free water was used (AG00021000, 2114 BATCH 15595401, Sharlau, Spain) as no-template control.

Statistical analysis. Data is expressed as mean \pm standard deviation (SD) for variables with Gaussian distribution and as median (25th-75th percentile) for variables without normal distribution. Normality of continuous data was assessed using the Kolmogorov-Smirnov test, analysis of the Q-Q plots and distribution in the histograms. Normal distribution was assessed and if necessary variables were natural-log transformed (ln). We specifically used a log transformation for NT-pro-BNP, TSH, and rT3 parameters. 


\begin{tabular}{|c|c|c|c|c|c|}
\hline Gene/chromosome location & Polymorphism ID & Function & Variation & MAF $^{*}$ & MAF \\
\hline \multirow[t]{3}{*}{ DIO1/1p32.3 } & rs11206244 & 3'UTR & c. $* 29 \mathrm{C}>\mathrm{T}$ & $\mathrm{T}=0.313$ & $\mathrm{~T}=0.348$ \\
\hline & rs12095080 & 3'UTR & c.* $1058 \mathrm{~A}>\mathrm{G}$ & $\mathrm{G}=0.093$ & $G=0.081$ \\
\hline & rs2235544 & int3 & c. $682-34 \mathrm{C}>\mathrm{A}$ & $\mathrm{A}=0.460$ & $\mathrm{~A}=0.481$ \\
\hline \multirow[t]{2}{*}{ DIO2/14q31.1 } & rs225014 & missense, 3'UTR & p.Thr92Ala & $\mathrm{C}=0.458$ & $\mathrm{C}=0.279$ \\
\hline & rs225015 & 3'UTR & c. $* 1453 \mathrm{C}>\mathrm{T}$ & $\mathrm{A}=0.443$ & $\mathrm{~A}=0.260$ \\
\hline DIO3/14q32.31 & rs945006 & 3'UTR & c. $* 529 \mathrm{~T}>\mathrm{G}$ & $\mathrm{G}=0.189$ & $\mathrm{G}=0.066$ \\
\hline
\end{tabular}

Table 1. General information about genotyped loci for DIO1, DIO2 and DIO3 polymorphisms. DIO deiodinases, $\mathrm{MAF}^{\ddagger}$ - reported minor allele frequencies in single nucleotide polymorphisms databases from 1000 Genome Phase III combined population (http://www.ncbi.nlm.nih.gov/snp), MAF - minor allele frequencies in the present cohort, UTR - untranslated region; int - intron.

Each SNP was tested for Hardy-Weinberg equilibrium (HWE) http://ihg.gsf.de/cgi-bin/hw/hwal.pl ${ }^{55}$, in case and contro l populations, using the Chi-square test or the Fisher's exact test before inclusion in the association statistics ( $p>0.01$ threshold). Baseline clinical characteristics, TH levels, fT3 ranges ( $1^{\text {st }}$ quartile versus $\geq 2^{\text {nd }}$ quartile of fT3), NT-pro-BNP, and DIO1, DIO2, DIO3 genotypes were compared across the cardiac-related death and survivors groups. Student's t, Mann-Whitney's U, Chi-square or Fisher's exact tests were used to compare group scores as appropriate. Correlations between fT3, NT-pro-BNP were assessed using Pearson product-moment analysis (Pearson $\mathrm{r}$ ). A p value $<0.05$ (two-tailed) was regarded as significant.

Univariate and multivariable Cox regression analyses were used to assess hazard ratio [HR] for all-cause and cardiac mortality. We made stringent attempts to control for the potentially confounding effect of (ln) NT-pro-BNP and other relevant sociodemographic and clinical factors such as age, Killip class, history of MI, history of hypertension, history of diabetes mellitus, history of chronic pulmonary disease and ST-elevation myocardial infarction. Kaplan-Meier survival curves for cardiac-related death and a log-rank (Mantel-Cox) test were employed for the analysis of survival curves. Statistical analyses was performed using the Statistical Package for the Social Science (SPSS23) for Windows.

\section{Results}

Baseline clinical characteristics, biomarkers levels and outcomes. Baseline demographics, clinical characteristics, CAD risk factors, concomitant disease, current treatment and concentration of biomarkers of 290 AMI patients are shown in Table 2. Two hundred and twenty four patients (77\%) had AMI with ST-elevation, $66(23 \%)$ with non ST-elevation, $236(82 \%)$ had AH, 55 (19\%) with DM and nine patients (3\%) had chronic pulmonary disease. The majority of patients were Killip class II (74\%), Killip class III (5\%), and Killip class IV (3\%). The mean of LVEF was $42.6 \pm 9.8 \%$. Eighty one percent of patients were taking beta-blockers, $92 \%$ - platelet antiaggregants, $89 \%$ - angiotensin-converting-enzyme inhibitors, and $10 \%$ - diuretics, and other medications.

During the two-year follow-up period there were a total of 14 cardiac-related and 21 all-cause deaths. Patients in the cardiac-related death group were older, with more frequent cases of previous MI, a higher Killip class, a higher level of NT-pro-BNP, and more frequent cases of first quartile fT3 levels, as compared to survivors (Table 3). As well, there was a trend between first quartile of fT 3 and higher cardiac-related mortality rates during first 30-days after a cardiac event (data not shown): patients with first quartile of fT3 consisted of older women with more severe HF (Killip class $>$ I), followed by more cases of DM, higher NT-pro-BNP and troponin I levels, lower T3, reduced hemoglobin and hematocrit levels. Negative associations between fT3 and NT-pro-BNP $(\mathrm{r}=-0.30, \mathrm{p}<0.001)$ were established.

Association between deiodinases gene polymorphisms and cardiac mortality. Genotype distributions of all SNPs were found to be in $\operatorname{HWE}(\mathrm{p}=0.203$ for $\mathrm{rs} 11206244-\mathrm{C} / \mathrm{T}, \mathrm{p}=0.457$ for rs $12095080-\mathrm{A} / \mathrm{G}$, $\mathrm{p}=0.105$ for $\mathrm{rs} 2235544-\mathrm{A} / \mathrm{C}, \mathrm{p}=0.492$ for $\mathrm{rs} 225014-\mathrm{T} / \mathrm{C}, \mathrm{p}=0.677$ for $\mathrm{rs} 225015-\mathrm{G} / \mathrm{A}, \mathrm{p}=0.226$ for rs $945006-\mathrm{T} / \mathrm{G})$. A relationship between gene polymorphisms and mortality was made in both cardiac mortality and survivor patient groups. Associations between DIO1 (rs11206244-C/T, rs12095080-A/G and rs2235544-A/C), DIO2 (rs225014-T/C, rs225015-G/A), and DIO3 (rs945006-T/G) gene variants and cardiac mortality showed that in a case of assessed DIO2, DIO3 polymorphisms, none of the SNPs were significantly associated with cardiac mortality in this AMI cohort.

However, the DIO1 gene rs12095080 heterozygous AG genotype $(\mathrm{OR}=3.97 ; 95 \% \mathrm{CI}=1.45-10.89 ; \mathrm{p}=0.005)$ showed a significant increased risk for cardiac-related mortality, while the major wild type homozygous AA genotype $(\mathrm{OR}=0.26 ; 95 \% \mathrm{CI}=0.09-0.71 ; \mathrm{p}=0.006)$ was linked to increased survival. Allele analysis revealed that mutant $\mathrm{G}$ allele was significantly associated $(\mathrm{OR}=3.31 ; 95 \% \mathrm{CI}=1.27-8.61 ; \mathrm{p}=0.036)$ with the risk of two year cardiac mortality (Table 4).

The prognostic importance of clinical variables, thyroid hormones, NT-pro-BNP and deiodinase genotypes on the mortality. Univariate regression analysis indicated that age, Killip class, NT-pro-BNP and history of chronic pulmonary disease were associated with all-cause mortality. The multiple Cox regression model showed no significant predictors of all-cause mortality (Table 5).

Univariate regression analysis indicated that age, Killip class, previous MI, NT-pro-BNP, history of chronic pulmonary disease as well as first quartile versus $\geq$ second quartile of fT3 and DIO1 gene rs12095080 were 


\begin{tabular}{|c|c|}
\hline Characteristics & $\mathrm{N}=290$ \\
\hline Age (years), mean $\pm S D$ & $62.0 \pm 11.4$ \\
\hline Body mass index, mean $\pm \mathrm{SD}$ & $29.9 \pm 17.8$ \\
\hline Systolic pressure $(\mathrm{mmHg})$, mean $\pm \mathrm{SD}$ & $141.8 \pm 25.9$ \\
\hline Diastolic pressure $(\mathrm{mmHg})$, mean $\pm \mathrm{SD}$ & $82.5 \pm 13.5$ \\
\hline \multicolumn{2}{|l|}{ Gender, n (\%): } \\
\hline Men & $209(72.1)$ \\
\hline Women & $81(27.9)$ \\
\hline \multicolumn{2}{|l|}{ Acute myocardial infarction type, n (\%): } \\
\hline With ST-segment elevation & $224(77.2)$ \\
\hline Non ST-segment elevation & $66(22.8)$ \\
\hline \multicolumn{2}{|l|}{ Myocardial infarction number, $\mathrm{n}(\%)$ : } \\
\hline First & $246(84.8)$ \\
\hline Previous & $44(15.2)$ \\
\hline \multicolumn{2}{|l|}{ Killip class, $\mathrm{n}(\%)$ : } \\
\hline I & $53(18.3)$ \\
\hline II & $214(73.8)$ \\
\hline III & $15(5.2)$ \\
\hline IV & $8(2.7)$ \\
\hline History of hypertension, n (\%) & $236(81.6)$ \\
\hline History of diabetes mellitus, $\mathrm{n}(\%)$ & $55(19.0)$ \\
\hline History of chronic pulmonary disease, n (\%) & $9(3.1)$ \\
\hline Coronary Angioplasty and Stenting, n (\%) & $240(82.8)$ \\
\hline \multicolumn{2}{|l|}{ Medications } \\
\hline Nitrate, n (\%) & $238(82.1)$ \\
\hline Beta-blockers, n (\%) & $235(81.0)$ \\
\hline ACE inhibitors, n (\%) & $258(89.0)$ \\
\hline Diuretics, n (\%) & $29(10.0)$ \\
\hline Antiplatelet, n (\%) & $267(92.1)$ \\
\hline Statins, n (\%) & $264(91.0)$ \\
\hline Insulin therapy, $\mathrm{n}(\%)$ & $22(7.6)$ \\
\hline $\begin{array}{l}\text { N-terminal pro-B-Type natriuretic peptide } \\
\text { (pg/mL), median (interquartile ranges) }\end{array}$ & $1330.0(489.0-3461.0)$ \\
\hline $\begin{array}{l}\text { Thyroid-stimulating hormone (mIU/l), median } \\
\text { (interquartile ranges) }\end{array}$ & $1.00(0.6-1.5)$ \\
\hline Free Thyroxine (pmol/l), mean $\pm \mathrm{SD}$ & $16.8 \pm 2.7$ \\
\hline Free Triiodothyronine (pmol/mL), mean $\pm \mathrm{SD}$ & $4.4 \pm 0.7$ \\
\hline $\begin{array}{l}\text { Reverse Triiodothyronine }(\mathrm{pg} / \mathrm{mL}) \text {, median } \\
\text { (interquartile ranges) }\end{array}$ & $646.9(489.5-1473.5)$ \\
\hline Total Triiodthyronine (nmol/l), mean \pm SD & $1.6 \pm 0.3$ \\
\hline
\end{tabular}

Table 2. Sociodemographic, clinical characteristics and biomarkers of patients with acute myocardial infarction. Values are presented as the mean $\pm \mathrm{SD}$, median (interquartile range), or percentage.

all significantly associated with cardiac-related mortality. Furthermore, after adjustment for clinical and demographic variables, the multiple Cox regression model showed that NT-pro-BNP $(\mathrm{HR}=2.11 ; 95 \% \mathrm{CI}=1.18-3.78$; $\mathrm{p}=0.012)$ and first quartile of $\mathrm{fT} 3$, and DIO1 gene rs 12095080 are significant risk factors for cardiac-related mortality $(\mathrm{HR}=1.74 ; 95 \% \mathrm{CI}=1.04-2.91 ; \mathrm{p}=0.034)$ after AMI (Table 5).

Kaplan-Meier two-year survival curves stratified on fT3 levels, according quartiles, provided significant prognostic information. The highest risk for cardiac mortality was among AMI patients within the first quartile of fT3, compared to patients with all other quartiles $(\mathrm{HR}=3.57 ; 95 \% \mathrm{CI}=1.20-10.62 ; \mathrm{p}=0.022)$ (Fig. 1 ). Moreover, Kaplan-Meier analyses showed decreased length of survival in a group of DIO1 gene rs12095080 AG genotype carriers $(\mathrm{HR}=4.09 ; 95 \% \mathrm{CI}=1.42-11.78 ; \mathrm{p}=0.009)$ (Fig. 2). Patients carrying rs 12095080 heterozygous genotype were found to experience death 2.5 months earlier (19.7 \pm 1.0 months vs. $22.2 \pm 0.23$ months; log-rank $\chi^{2}=7.99, \mathrm{p}=0.005$ ), as compared to AA genotype carriers (Fig. 2).

\section{Discussion}

In this research study we aimed to explore possible associations between serum levels of $\mathrm{TH}$, genetic polymorphisms of DIO, and NT-pro-BNP with long-term outcomes in AMI patients.

It was found that lower fT3 levels, DIO1 gene rs12095080, as well as higher NT-pro-BNP on admission are all associated with cardiac-related mortality after AMI. The hypothesis proposing that 


\begin{tabular}{|c|c|c|c|}
\hline \multirow[b]{2}{*}{ Characteristics } & \multirow{2}{*}{\begin{tabular}{|l|} 
Cardiac death \\
$\mathrm{n}=14$
\end{tabular}} & \multirow{2}{*}{\begin{tabular}{|l|} 
Survived \\
$\mathrm{n}=269$
\end{tabular}} & \multirow[b]{2}{*}{ p-value } \\
\hline & & & \\
\hline Age (years), mean $\pm S D$ & $69.6 \pm 8.4$ & $61.3 \pm 11.3$ & 0.003 \\
\hline Body mass index, mean $\pm \mathrm{SD}$ & $30.7 \pm 4.2$ & $29.9 \pm 18.3$ & 0.603 \\
\hline Gender, n (\%): & & & 0.548 \\
\hline Men & $9(64.3)$ & $194(72.1)$ & \\
\hline Women & $5(35.7)$ & $75(27.9)$ & \\
\hline Myocardial infarction classification, n (\%): & & & 0.205 \\
\hline ST-elevation myocardial infarction & $9(64.3)$ & $211(78.4)$ & \\
\hline Non-ST elevation myocardial infarction & $5(35.7)$ & $58(21.6)$ & \\
\hline \multicolumn{4}{|l|}{ Myocardial infarction number, n (\%): } \\
\hline First & $9(64.3)$ & $230(85.5)$ & 0.049 \\
\hline Previous & $5(35.7)$ & $39(14.5)$ & \\
\hline Killip class, n (\%): & & & 0.004 \\
\hline I & $1(7.1)$ & $51(19.0)$ & \\
\hline II & $8(57.1)$ & $202(75.1)$ & \\
\hline III & $4(28.6)$ & $11(4.1)$ & \\
\hline IV & $1(7.1)$ & $5(1.9)$ & \\
\hline Hystory of Hypertension, n (\%) & $13(92.9)$ & $217(80.7)$ & 0.480 \\
\hline History of Diabetes mellitus, n (\%) & $5(35.7)$ & $48(17.8)$ & 0.149 \\
\hline History of Previous stroke, n (\%) & $2(14.3)$ & $11(4.1)$ & 0.130 \\
\hline History of Chronic renal disease, $\mathrm{n}(\%)$ & $1(7.1)$ & $11(4.1)$ & 0.463 \\
\hline History of Chronic pulmonary disease, n (\%) & $2(14.3)$ & $6(2.2)$ & 0.054 \\
\hline $\begin{array}{l}\text { N-terminal pro-B-Type natriuretic peptide }(\mathrm{pg} / \mathrm{mL}) \text {, median } \\
\text { (interquartile ranges) }\end{array}$ & $5104.0(1648.5-13863.0)$ & $1238.0(475.0-3191.0)$ & $<0.001$ \\
\hline $\begin{array}{l}\text { Thyroid-stimulating hormone (mIU/l), median (interquartile } \\
\text { ranges) }\end{array}$ & $1.1(0.5-2.4)$ & $1.0(0.6-1.5)$ & 0.773 \\
\hline Free Thyroxine (pmol/l), mean $\pm \mathrm{SD}$ & $17.4 \pm 3.5$ & $16.8 \pm 2.6$ & 0.579 \\
\hline Free Triiodothyronine (pmol $/ \mathrm{mL})$, mean $\pm \mathrm{SD}$ & $4.1 \pm 0.8$ & $4.4 \pm 0.7$ & 0.195 \\
\hline $\begin{array}{l}1^{\text {st }} \text { quartile of Free Triiodothyronine versus } \geq 2^{\text {nd }} \text { quartile of } \\
\text { Free Triiodothyronine, } n(\%):\end{array}$ & & & 0.021 \\
\hline $1^{\text {st }}$ quartile of Free triiodothyronine & $7(58.3)$ & $63(23.4)$ & \\
\hline$\geq 2^{\text {nd }}$ quartile of Free triiodothyronine & $6(46.2)$ & $206(76.6)$ & \\
\hline $\begin{array}{l}\text { Reverse Triiodothyronine }(\mathrm{pg} / \mathrm{mL}) \text {, median (interquartile } \\
\text { ranges) }\end{array}$ & $941.8(329.5-1858.7)$ & 635.6(491.5-1451.7) & 0.849 \\
\hline Total Triiodthyronine (nmol/l), mean $\pm \mathrm{SD}$ & $1.6 \pm 0.4$ & $1.6 \pm 0.3$ & 0.855 \\
\hline
\end{tabular}

Table 3. Clinical characteristics of patients, who experienced cardiac death or survived due to MI. Values are presented as the mean $\pm \mathrm{SD}$, median (interquartile range), or percentage. p-values are presented for Student's $\mathrm{t}$ test, Mann-Whitney's U test, the Chi-square test or Fisher's exact test as appropriate.

variations in TH concentrations within the statistically normal range may influence disease outcomes is not entirely new ${ }^{26,56,57}$. Nevertheless, a low T3 syndrome does not only reflect AMI status, but it has also been documented in a number of other disorders ${ }^{58-61}$.

Independent of time-course, type and severity, a low T3 state may serve as an adaptive mechanism which reduces metabolic demands by reducing the catabolic processes of the disease ${ }^{8}$. A low T3 syndrome was a frequent finding in patients with cardiac pathology and without a history of thyroid dysfunction, particularly among patients with HF, AMI, and those following cardiac surgery ${ }^{15-17,62-65}$. However, the exact point of occurrence of THs alterations, after an ACS, is not clearly understood ${ }^{2,66-68}$. Timing of TH alterations is still debated topic in the scientific literature. However, most of the studies agree that the first five days of ACS are the most crucial for changes in T3 and rT3. Iltumur et al. ${ }^{69}$ observed that patients with complicated MI (caused by ischemia) have a lower total and fT3. Besides, patients with prolonged cardiac arrest showed lower total T3 and fT3 levels than those with shorter one. Furthermore, during the AMI stage, drugs like nonsteroidal anti-inflammatory agents, aspirin, heparin and furosemide ( $>80 \mathrm{mg} /$ day) might have an effect of displacing T4 and T3 from TH binding sites on TH binding proteins, which modify hormone delivery to the location of its use ${ }^{70,71}$.

Our study findings correspond to the findings of Zhang et al. ${ }^{17}$ exemplifying that patients with AMI and with first quartile of fT3 levels, are more likely to be older women, with severe HF (Killip class >I), followed by DM. Our study AMI patients also had a higher level of troponin I, lower T3, as well as lower hemoglobin and hematocrit levels. The low T3 pattern pathophysiological role is not well understood, although high mortality among patients with low T3 levels is found in numerous studies ${ }^{1,12,17,37,63}$. Conversely, other studies have not discovered an independent prognostic role for low T3 levels in cardiovascular patients ${ }^{72-75}$. Our study revealed a decreased length of survival in AMI patients with first quartile of fT3, confirming previous findings. Additionally, we estimate that fT3 levels within the normal concentration ranges was probably due to omitted analysis of TH during the later post-AMI period when greater fT3 downregulations could be observed ${ }^{2,16,18,66-68,76}$. 


\begin{tabular}{|c|c|c|c|c|c|c|c|}
\hline \multirow[b]{2}{*}{ Gene } & \multirow[b]{2}{*}{ SNPs } & \multirow{2}{*}{\begin{tabular}{|l|}
$\begin{array}{l}\text { Cardiac } \\
\text { death }\end{array}$ \\
$\mathrm{n}=14$ \\
\end{tabular}} & \multirow{2}{*}{$\begin{array}{l}\text { Survived } \\
\mathrm{n}=269\end{array}$} & \multirow[b]{2}{*}{$x^{2}$} & \multirow[b]{2}{*}{ OR } & \multirow[b]{2}{*}{ 95\% CI } & \multirow[b]{2}{*}{ p-value } \\
\hline & & & & & & & \\
\hline \multirow{18}{*}{ DIO1 } & $r s 11206244$ & & & 0.437 & & & 0.803 \\
\hline & $\mathrm{CC}$ & $7(50.0 \%)$ & $111(41.4 \%)$ & 0.403 & 1.390 & $0.501-3.857$ & 0.526 \\
\hline & \begin{tabular}{|l|}
$\mathrm{CT}$ \\
\end{tabular} & $6(42.9 \%)$ & $130(48.5 \%)$ & \begin{tabular}{|l|}
0.170 \\
\end{tabular} & 0.805 & $0.287-2.261$ & 0.680 \\
\hline & TT & $1(7.1 \%)$ & $27(10.1 \%)$ & 0.128 & 0.698 & $0.095-5.136$ & 1.000 \\
\hline & C allele & 0.71 & 0.66 & 0.39 & 1.307 & $0.565-3.024$ & 0.531 \\
\hline & T allele & 0.29 & 0.34 & 0.39 & 0.756 & $0.331-1.771$ & 0.531 \\
\hline & $r s 12095080$ & & & 8.027 & & & 0.065 \\
\hline & AA & $8(57.1 \%)$ & $229(85.1 \%)$ & 7.657 & 0.259 & $0.094-0.711$ & 0.006 \\
\hline & AG & $6(42.9 \%)$ & $39(14.5 \%)$ & 8.003 & 3.967 & $1.446-10.885$ & 0.005 \\
\hline & GG & 0 & $1(0.4 \%)$ & 0.052 & - & - & 1.000 \\
\hline & A allele & 0.79 & 0.92 & 6.66 & 0.302 & $0.116-0.788$ & 0.036 \\
\hline & G allele & 0.21 & 0.08 & 6.66 & 3.306 & $1.269-8.610$ & 0.036 \\
\hline & $r s 2235544$ & & & 3.162 & & & 0.226 \\
\hline & AA & $1(7.1 \%)$ & $66(24.7 \%)$ & 2.263 & 0.246 & $0.003-1.844$ & 0.200 \\
\hline & $\mathrm{AC}$ & $8(57.1 \%)$ & $146(54.7 \%)$ & 0.033 & 1.100 & $0.392-3.086$ & 0.857 \\
\hline & $\mathrm{CC}$ & $5(35.7 \%)$ & $55(20.6 \%)$ & 1.810 & 2.046 & $0.712-5.879$ & 0.179 \\
\hline & A allele & 0.36 & 0.52 & 2.85 & 0.512 & $0.232-1.129$ & 0.092 \\
\hline & C allele & 0.64 & 0.48 & 2.85 & 1.955 & $0.886-4.313$ & 0.092 \\
\hline \multirow{12}{*}{$\mathrm{DIO} 2$} & rs225014 & & & 2.899 & & & 0.248 \\
\hline & TT & $10(71.4 \%)$ & $134(49.8 \%)$ & 2.488 & 2.413 & $0.775-7.514$ & 0.170 \\
\hline & TC & $4(28.6 \%)$ & $115(42.8 \%)$ & 1.098 & 0.551 & $0.177-1.716$ & 0.408 \\
\hline & CC & 0 & $20(7.4 \%)$ & 1.120 & - & - & 0.609 \\
\hline & T allele & 0.86 & 0.71 & 2.78 & 2.428 & $0.829-7.113$ & 0.095 \\
\hline & C allele & 0.14 & 0.29 & 2.78 & 0.412 & $0.141-1.206$ & 0.095 \\
\hline & $r s 225015$ & & & 2.209 & & & 0.312 \\
\hline & GG & $10(71.4 \%)$ & $143(53.2 \%)$ & \begin{tabular}{|l|}
1.788 \\
\end{tabular} & 2.124 & $0.682-6.613$ & 0.181 \\
\hline & GA & $4(28.6 \%)$ & $108(40.1 \%)$ & 0.746 & 0.611 & $0.196-1.900$ & 0.577 \\
\hline & AA & 0 & $18(6.7 \%)$ & 1.000 & - & - & 0.610 \\
\hline & G allele & 0.86 & 0.73 & 2.15 & 2.193 & $0.748-6.429$ & 0.143 \\
\hline & A allele & 0.14 & 0.27 & 2.15 & 0.456 & $0.156-1.337$ & 0.143 \\
\hline \multirow{6}{*}{ DIO3 } & rs 945006 & & & 0.009 & & & 1.000 \\
\hline & TT & $12(85.7 \%)$ & $233(86.6 \%)$ & 0.009 & 0.931 & $0.217-3.997$ & 0.923 \\
\hline & TG & $2(14.3 \%)$ & $36(13.4 \%)$ & \begin{tabular}{|l|}
0.009 \\
\end{tabular} & 1.075 & $0.250-4.615$ & 1.000 \\
\hline & GG & 0 & 0 & - & - & - & - \\
\hline & T allele & 0.93 & 0.93 & 0.01 & 0.932 & $0.213-4.085$ & 0.812 \\
\hline & G allele & 0.07 & 0.07 & 0.01 & 1.073 & $0.245-4.700$ & 0.812 \\
\hline
\end{tabular}

Table 4. Association between deiodinases genotype and two year cardiac-related mortality. DIO - deiodinases, SNP - single nucleotide polymorphism. Values are presented as number (percentage). p-values are presented for Hardy-Weinberg equilibrium (HWE) test and the Chi-square test or Fisher's exact test as appropriate. Bold values: $\mathrm{p}$-value $<0.05$ was regarded as significant. Source: HWE: www.had2know.com/academics/hardyweinberg-equilibrium-calculator.

The present study lends support to the theory advanced by other research teams that fT3 represents the biologically active form of TH, so an isolated reduction in its level could constitute a model of abnormal TH metabolism acting as a risk factor for $\mathrm{CAD}^{3,27-29}$. Further, subclinical hypothyroidism, characterized by normal serum concentrations of fT4 and elevated TSH showed as a predictor of atherosclerosis and MI risk in elderly women ${ }^{3,27,77,78}$. It is suggested that even within the clinically normal range variations of TH indicate abnormal TH metabolism associated with coronary disease risk and outcomes ${ }^{24,27-30,79}$. However, Ertas et al. ${ }^{28}$ showed that within the normal range fT3 levels were inversely associated with CAD severity. It was also found that lower fT3 concentrations independently predicted the severity of $\mathrm{CAD}^{29}$. Mayer et al., showed that even minor changes of fT4 may relate with severity of $\mathrm{HF}^{30,31}$. fT4 serum concentration levels association with coronary disease severity was also examined in Jung et al's study ${ }^{26}$. When compared with survivors patients that died within seven days after AMI had a higher fT4 level, thus it is possible to make an assumption that higher levels of fT4 might be associated with increased survival rate $^{2,25}$. Our present and previous studies and those of others, indicate association between fT3 or low-T3 syndrome with elevated NT-pro-BNP levels. This is a traditional predictor of poor prognoses in patients with AMI, indicating that a lower fT3 level would be a predictor of a poor prognosis in CAD and AMI patients ${ }^{17,23,80,81}$. The current study also presented a negative association between fT3, NT-pro-BNP levels and CAD outcomes which was confirmed by others authors ${ }^{80-83}$. 


\begin{tabular}{|c|c|c|c|c|}
\hline \multirow[b]{2}{*}{ Variable } & \multicolumn{2}{|l|}{ Univariate } & \multicolumn{2}{|l|}{ Multivariable } \\
\hline & HR (95\% CI) & p-value & HR $(95 \% \mathrm{CI})$ & p-value \\
\hline \multicolumn{5}{|l|}{ All-cause mortality } \\
\hline Age & $1.09(1.04-1.13)$ & $<0.001$ & $1.04(0.99-1.10)$ & 0.097 \\
\hline Killip class & $2.90(1.72-4.90)$ & $<0.001$ & $1.74(0.79-3.82)$ & 0.167 \\
\hline Previous myocardial infarction & $1.79(0.65-4.88)$ & 0.258 & $0.92(0.28-2.99)$ & 0.886 \\
\hline (ln) N-terminal pro-B-Type natriuretic peptide & $1.92(1.35-2.72)$ & $<0.001$ & $1.45(0.93-2.26)$ & 0.104 \\
\hline History of hypertension & $2.25(0.52-9.67)$ & 0.275 & $1.18(0.26-5.42)$ & 0.837 \\
\hline History of diabetes mellitus & $2.24(0.90-5.55)$ & 0.082 & $1.48(0.50-4.41)$ & 0.480 \\
\hline History of chronic pulmonary disease & $5.89(1.73-50.01)$ & 0.005 & $2.57(0.55-12.06)$ & 0.233 \\
\hline ST-elevation myocardial infarction & $2.12(0.88-5.10)$ & 0.096 & $1.81(0.69-4.79)$ & 0.230 \\
\hline Free Thyroxine & $1.00(0.85-1.18)$ & 0.987 & $0.88(0.75-1.02)$ & 0.096 \\
\hline Free Triiodothyronine & $0.55(0.26-1.18)$ & 0.124 & $0.69(0.34-1.41)$ & 0.691 \\
\hline $\begin{array}{l}1^{\text {st }} \text { quartile of Free Triiodothyronine versus } \geq 2^{\text {nd }} \text { quartile of } \\
\text { Free Triiodothyronine }\end{array}$ & $2.07(0.85-5.07)$ & 0.111 & $1.57(0.58-4.26)$ & 0.371 \\
\hline DIO1rs12095080 & $0.45(0.17-1.16)$ & 0.096 & $0.52(0.19-1.46)$ & 0.214 \\
\hline rs12095080 AG & $2.23(0.87-5.75)$ & 0.096 & $1.94(0.69-5.47)$ & 0.211 \\
\hline rs12095080 AA & $0.45(0.17-1.16)$ & 0.096 & $0.52(0.18-1.46)$ & 0.212 \\
\hline $\begin{array}{l}1^{\text {st }} \text { quartile of Free Triiodothyronine versus } \geq 2^{\text {nd }} \text { quartile of } \\
\text { Free Triiodothyronine \& rs12095080 }\end{array}$ & $1.66(1.09-2.51)$ & 0.018 & $1.41(0.90-2.20)$ & 0.131 \\
\hline \multicolumn{5}{|l|}{ Cardiac-related mortality } \\
\hline Age & $1.07(1.02-1.13)$ & 0.008 & $1.01(0.95-1.08)$ & 0.668 \\
\hline Killip class & $2.94(1.53-5.67)$ & 0.001 & $1.79(0.71-4.48)$ & 0.217 \\
\hline Previous myocardial infarction & $3.06(1.02-9.14)$ & 0.045 & $1.43(0.40-5.17)$ & 0.582 \\
\hline (ln) N-terminal pro-B-Type natriuretic peptide & $2.37(1.50-3.74)$ & $<0.001$ & $2.11(1.18-3.78)$ & 0.012 \\
\hline History of hypertension & $3.09(0.40-23.61)$ & 0.277 & $1.47(0.18-12.20)$ & 0.722 \\
\hline History of diabetes mellitus & $2.50(0.84-7.46)$ & 0.101 & $2.11(0.59-7.58)$ & 0.252 \\
\hline History of chronic pulmonary disease & $6.39(1.43-28.58)$ & 0.015 & $3.61(0.61-21.30)$ & 0.157 \\
\hline ST-elevation myocardial infarction & $1.97(0.66-5.87)$ & 0.226 & $2.27(0.65-7.95)$ & 0.199 \\
\hline Free Thyroxine & $1.08(0.89-1.29)$ & 0.446 & $1.01(0.95-1.08)$ & 0.652 \\
\hline Free Triiodothyronine & $0.43(0.16-1.14)$ & 0.089 & $0.82(0.28-2.36)$ & 0.547 \\
\hline $\begin{array}{l}1^{\text {st }} \text { quartile of Free Triiodothyronine versus } \geq 2^{\text {nd }} \text { quartile of } \\
\text { Free Triiodothyronine }\end{array}$ & $3.57(1.20-10.62)$ & 0.022 & $2.30(0.68-7.73)$ & 0.180 \\
\hline DIO1rs12095080 & $0.25(0.09-0.71)$ & 0.009 & $0.32(0.10-1.09)$ & 0.069 \\
\hline rs12095080 AG & $4.09(1.42-11.78)$ & 0.009 & $3.14(0.92-10.72)$ & 0.069 \\
\hline rs12095080 AA & $0.25(0.09-0.71)$ & 0.009 & $0.32(0.09-1.09)$ & 0.069 \\
\hline $\begin{array}{l}1^{\text {st }} \text { quartile of Free Triiodothyronine versus } \geq 2^{\text {nd }} \text { quartile of } \\
\text { Free Triiodothyronine \& rs12095080 }\end{array}$ & $2.40(1.48-3.92)$ & $<0.001$ & $1.74(1.04-2.91)$ & 0.034 \\
\hline
\end{tabular}

Table 5. Cox regression analysis for factors associated with all-cause and cardiac-related mortality. aMultiple Cox regression analyses adjusted for age, Killip class, previous myocardial infarction, (ln) N-terminal pro-BType natriuretic peptide, history of hypertension, history of diabetes mellitus, history of chronic pulmonary disease, ST-elevation myocardial infarction.

There are several well-known TH-pathway genes such as DIO, TSH receptor (THR), and TH transporters (SLCO, MCT), which have been associated with TH levels ${ }^{84}$. Variants in both DIO1 and DIO2 genes were recently reported to alter TH levels in healthy individuals ${ }^{34,45,85,86}$. TH metabolism roles are determined by three iodothyronine deiodinases DIO1, DIO2 and DIO3 encoded by a separate gene ${ }^{37,38,40,87}$. The DIO1, which is responsible for converting T4 into T3, and contributes to the local hypothyroid state in the failing heart ${ }^{4,12,37}$. It was shown, experimentally, that alterations in DIO1 and DIO2 promote cardiac activity of DIO3, converting T4 and T3 to inactive reverse $\mathrm{T} 3$ and diiodothyronine (T2) in rats following $\mathrm{MI}^{88}$. Altered thyroid homeostasis in patients with cardiovascular disorders could modify cardiac gene expression and contribute to impaired cardiac function ${ }^{89,90}$. A candidate gene study revealed rs2235544 in DIO1 gene was associated with higher fT3 and lower fT4 and rT3 levels in both patients receiving TH replacement therapy and in a large population of healthy individuals. Rare $\mathrm{C}$ allele was associated with improved DIO1 function ${ }^{44,52}$. Several studies identified rs11206244 in DIO1, which was also associated with fT4, rT3 and fT3 concentrations ${ }^{34,91}$. Numerous studies disclosed an association between DIO1, DIO2, DIO3 polymorphisms and fT3 and other TH levels ${ }^{33,34,42,92}$. Our data of the same cohort also endorsed that DIO1, DIO2 gene polymorphisms are mainly associated with T3, fT4, fT3/fT4, $(\ln ) \mathrm{rT} 3$ levels, while organic anion transporter polypeptide $1 C 1 \mathrm{rs} 1515777-\mathrm{AG}$ minor allele homozygous genotype was associated with a decrease in circulating fT3, fT3/fT4 in CAD patients after $\mathrm{AMI}^{46}$.

Genetic variations in deiodinases may affect multiple clinical endpoints ${ }^{36,37,42,93}$. It was shown that the development of CAD is the result of complex interactions between numerous environmental factors and genetic variants 


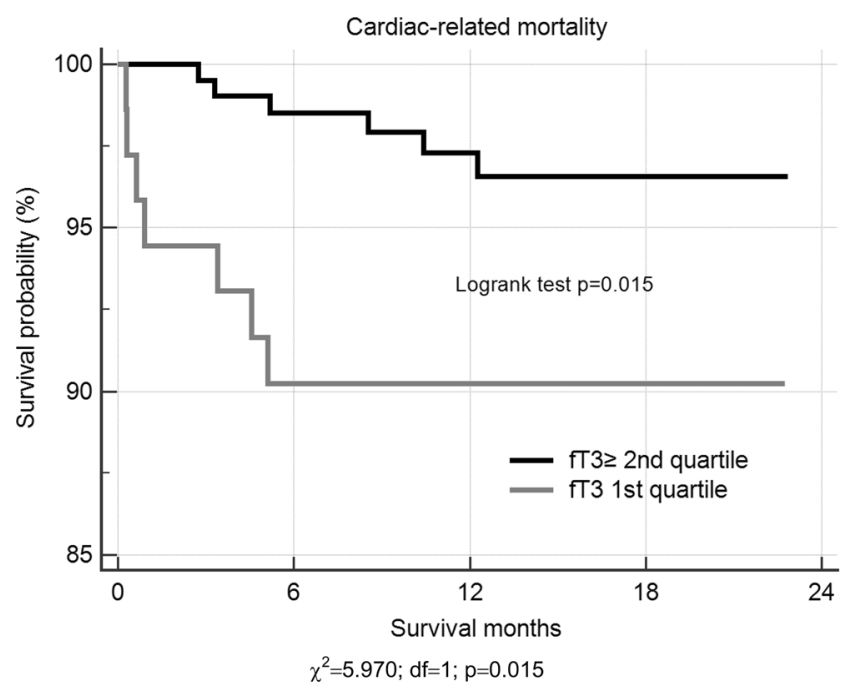

Figure 1. Two year Kaplan-Meier survival curves for cardiac-related death in patients with AMI stratified on fT3 quartiles. A log-rank test was used to compare survival curves.

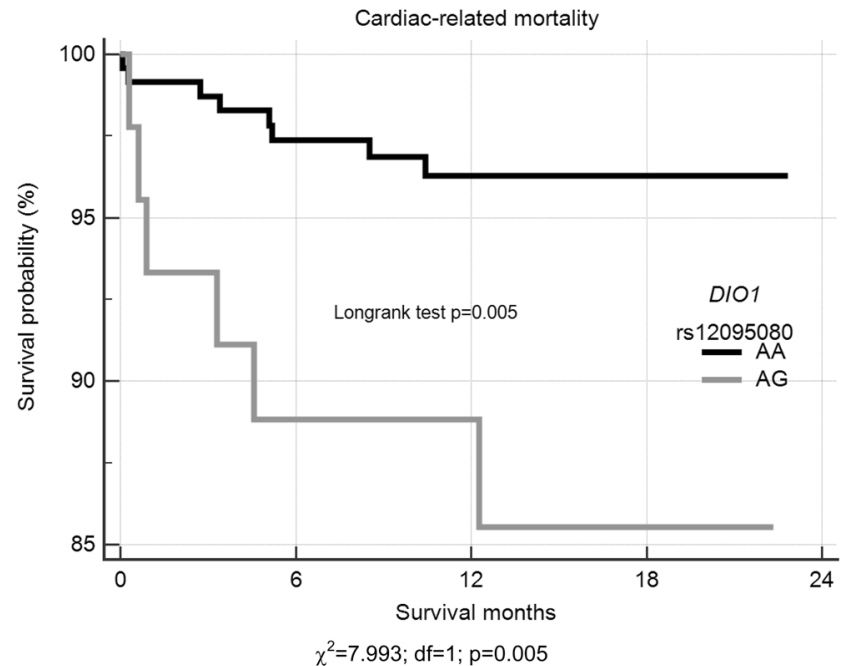

Figure 2. Two year Kaplan-Meier survival curves for cardiac-related death in patients with AMI according to D1O1rs12095080 genotypes. A log-rank test was used to compare survival curves.

at many loci ${ }^{94,95}$. In our previous study we found that DIO1 rs12095080 was associated with AH, while DIO2 rs225015 was associated with DM, and SNP rs974453-genotypes was associated with STEMI within the OATP1C1 gene $^{46}$.

Lee et al. found that cardiovascular mortality was higher in subjects with the rs4977574 GG genotype than in those with other genotypes ${ }^{96}$. The association between four SNPs on chromosome 9p21, CAD, and MI has been replicated several times in multiple populations ${ }^{97-100}$ In patients with MI with ST-segment elevation Szpakowicz et al. revealed association between the rs 12526453 of the phosphatase and actin regulator 1 (PHACTR1) gene and 5 -year mortality ${ }^{101}$. However, in another study, the DIO2 Thr92Ala polymorphism was not related with thyroid parameters, cognitive functioning and health-related quality of life ${ }^{102}$. In the present study we found a relationship between SNPs in DIO1 gene rs12095080 heterozygous genotype (AG) and cardiac-related mortality. It should be noted that no patients in the cardiac-related death group carried the homozygous mutant GG genotype of this SNPs. Patients carrying rs12095080 heterozygous genotype experienced 2.5 months shorter median survival as compared to AA genotype carriers. Our preliminary analysis shows that $\mathrm{G}$ allele could be a favourable variable to investigate for AMI patient's prognosis. To our knowledge, there are no reports showing the importance of fT3 ranges and genetic variability of DIO1 in the long-term outcomes of the patients with AMI. There is evidence that the G variant in rs12095080, identified in the 3' UTR of human DIO1 mRNA, is associated with higher T3/rT3 ratio in serum. This may suggest that some variants in this SNPs may result in increased DIO1 activity ${ }^{103}$. Palmer et al. ${ }^{104}$ showed that angiotensin-converting enzyme genotype powerfully predicted mortality in patients after 
AMI. They also showed that the ACE genotype DD was positively associated with the B type natriuretic peptide and was an independent predictor of death and the effects the response to treatment ${ }^{105}$.

To our knowledge this study is the first one to examine how concentrations of TH and genetic markers in patients after AMI might contribute to long term outcomes. However, our findings are still exploratory and it would be premature to use them as a basis for risk stratification in patients with CAD. For example, future studies are needed to explore fT3 and gene polymorphism mutual interaction on the underlying cardiovascular mortality mechanisms. Understanding the genetic factors contribution to $\mathrm{TH}$ expression that predict cardiac-related mortality may open new markers and treatment targets for management of cardiovascular disease. For example, as suggested by Pingitore et al. ${ }^{18}$ by knowing the exact mechanism we might not only measure fT 3 concentration in patients after an AMI and patients with multiple CAD risk factors but also treat those with low fT3 and see whether their clinical outcomes improve.

The main limitation of this study is that clinical research was performed in a single centre with a limited number of subjects. These results require validation in studies that replicate the model and include a higher number of cases and controls. Additionally, the majority of studied AMI patients had mild to moderate HF and we did not include other risk factors in our study, such as left ventricular ejection fraction or smoking. Thus, the results presented may be limited in their generalizability and may not apply to patients with more advanced HF.

Finally, baseline levels of TH were not evaluated in this study, as TH was measured only on admission to the ICU and was not investigated during the later post-AMI period when the hormone concentration decline is lasting $^{2,66-68}$. The strengths of this study include its novelty - the assessment of an impact of the fT3 ranges and TH gene polymorphisms on long-term mortality while controlling for disease severity and other CAD risk factors in patients with AMI.

\section{Conclusions}

Lower fT3 level and DIO1 gene rs12095080 as well as higher NT-pro-BNP on admission are associated with cardiac-related mortality after AMI. In a case of DIO1 gene rs12095080, heterozygous AG genotype was significantly associated with a higher risk for cardiac mortality. Conversely, major wild type homozygous AA genotype was linked to better survival within the two year follow-up period.

Ethics approval and consent to participate. The study and its consent procedures were approved by the Kaunas Regional Biomedical Research Ethics at Lithuanian University of Health Sciences, Kaunas, Lithuania and conform to the principles outlined in the Declaration of Helsinki. Written informed consent was obtained from each study patient.

\section{Data availability}

The datasets analysed during the current study are available from the corresponding author upon request

Received: 4 November 2019; Accepted: 28 April 2020;

Published online: 08 June 2020

\section{References}

1. Iervasi, G. et al. Low-T3 syndrome: a strong prognostic predictor of death in patients with heart disease. Circulation 107, 708-713 (2003).

2. Friberg, L., Werner, S., Eggertsen, G. \& Ahnve, S. Rapid down-regulation of thyroid hormones in acute myocardial infarction: is it cardioprotective in patients with angina? Archives of internal medicine 162, 1388-1394 (2002).

3. Coceani, M., Iervasi, G., Pingitore, A., Carpeggiani, C. \& L'Abbate, A. Thyroid hormone and coronary artery disease: from clinical correlations to prognostic implications. Clinical cardiology 32, 380-385, https://doi.org/10.1002/clc.20574 (2009).

4. Pingitore, A. et al. Triiodothyronine levels for risk stratification of patients with chronic heart failure. The American journal of medicine 118, 132-136, https://doi.org/10.1016/j.amjmed.2004.07.052 (2005).

5. Zhao, J. V. \& Schooling, C. M. Thyroid function and ischemic heart disease: a Mendelian randomization study. Scientific Reports 7, 8515, https://doi.org/10.1038/s41598-017-07592-z (2017).

6. Docter, R., Krenning, E. P., de Jong, M. \& Hennemann, G. The sick euthyroid syndrome: changes in thyroid hormone serum parameters and hormone metabolism. Clinical endocrinology 39, 499-518 (1993).

7. McIver, B. \& Gorman, C. A. Euthyroid sick syndrome: an overview. Thyroid: official journal of the American Thyroid Association 7 , 125-132, https://doi.org/10.1089/thy.1997.7.125 (1997).

8. De Groot, L. J. Dangerous dogmas in medicine: the nonthyroidal illness syndrome. The Journal of clinical endocrinology and metabolism 84, 151-164, https://doi.org/10.1210/jcem.84.1.5364 (1999).

9. Chopra, I. J. Nonthyroidal illness syndrome or euthyroid sick syndrome? Endocrine practice: official journal of the American College of Endocrinology and the American Association of Clinical Endocrinologists 2, 45-52, https://doi.org/10.4158/ep.2.1.45 (1996).

10. Wang, B. et al. Non-thyroidal illness syndrome in patients with cardiovascular diseases: A systematic review and meta-analysis. International journal of cardiology 226, 1-10, https://doi.org/10.1016/j.ijcard.2016.10.039 (2017).

11. Ascheim, D. D. \& Hryniewicz, K. Thyroid hormone metabolism in patients with congestive heart failure: the low triiodothyronine state. Thyroid: official journal of the American Thyroid Association 12, 511-515, https://doi.org/10.1089/105072502760143908 (2002).

12. Iervasi, G. et al. Association between increased mortality and mild thyroid dysfunction in cardiac patients. Archives of internal medicine 167, 1526-1532, https://doi.org/10.1001/archinte.167.14.1526 (2007).

13. Chuang, C. P., Jong, Y. S., Wu, C. Y. \& Lo, H. M. Impact of triiodothyronine and N-terminal pro-B-type natriuretic peptide on the long-term survival of critically ill patients with acute heart failure. The American journal of cardiology 113, 845-850, https://doi. org/10.1016/j.amjcard.2013.11.039 (2014).

14. Hamilton, M. A., Stevenson, L. W., Luu, M. \& Walden, J. A. Altered thyroid hormone metabolism in advanced heart failure. Journal of the American College of Cardiology 16, 91-95 (1990).

15. Damman, P., de Winter, R. J., Wallentin, L. \& Fox, K. A. Letter by Damman et al regarding articles, "Long-term cardiovascular mortality after procedure-related or spontaneous myocardial infarction in patients with non-ST-segment elevation acute coronary syndrome: a collaborative analysis of individual patient data from the FRISC II, ICTUS, and RITA-3 Trials (FIR)" and "American 
College of Cardiology/American Heart Association/European Society of Cardiology/World Heart Federation universal definition of myocardial infarction classification system and the risk of cardiovascular death: observations from the TRITON-TIMI 38 Trial (Trial to Assess Improvement in Therapeutic Outcomes by Optimizing Platelet Inhibition With Prasugrel-Thrombolysis in Myocardial Infarction 38)". Circulation 126, e136-137; author reply e138, doi:10.1161/circulationaha.112.102509 (2012).

16. Kimura, T. et al. Involvement of circulating interleukin-6 and its receptor in the development of euthyroid sick syndrome in patients with acute myocardial infarction. European journal of endocrinology 143, 179-184, https://doi.org/10.1530/eje.0.1430179 (2000).

17. Zhang, B., Peng, W., Wang, C., Li, W. \& Xu, Y. A low fT3 level as a prognostic marker in patients with acute myocardial infarctions. Internal medicine (Tokyo, Japan) 51, 3009-3015 (2012).

18. Pingitore, A., Chen, Y., Gerdes, A. M. \& Iervasi, G. Acute myocardial infarction and thyroid function: new pathophysiological and therapeutic perspectives. Annals of medicine 44, 745-757, https://doi.org/10.3109/07853890.2011.573501 (2012).

19. Pantos, C., Mourouzis, I. \& Cokkinos, D. V. New insights into the role of thyroid hormone in cardiac remodeling: time to reconsider? Heart failure reviews 16, 79-96, https://doi.org/10.1007/s10741-010-9185-3 (2011).

20. Wassen, F. W. et al. Induction of thyroid hormone-degrading deiodinase in cardiac hypertrophy and failure. Endocrinology 143, 2812-2815, https://doi.org/10.1210/endo.143.7.8985 (2002).

21. Jankauskiene, E. et al. Relationship between left ventricular mechanics and low free triiodothyronine levels after myocardial infarction: a prospective study. Internal and emergency medicine 11, 391-398, https://doi.org/10.1007/s11739-015-1370-x (2016).

22. Shanoudy, H. et al. Early manifestations of "sick euthyroid" syndrome in patients with compensated chronic heart failure. Journal of cardiac failure 7, 146-152, https://doi.org/10.1054/jcaf.2001.24665 (2001).

23. Brozaitiene, J., Mickuviene, N., Podlipskyte, A., Burkauskas, J. \& Bunevicius, R. Relationship and prognostic importance of thyroid hormone and $\mathrm{N}$-terminal pro-B-Type natriuretic peptide for patients after acute coronary syndromes: a longitudinal observational study. BMC cardiovascular disorders 16, 45, https://doi.org/10.1186/s12872-016-0226-2 (2016).

24. Cappola, A. R. \& Ladenson, P. W. Hypothyroidism and atherosclerosis. The Journal of clinical endocrinology and metabolism $\mathbf{8 8}$, 2438-2444, https://doi.org/10.1210/jc.2003-030398 (2003).

25. Friberg, L., Drvota, V., Bjelak, A. H., Eggertsen, G. \& Ahnve, S. Association between increased levels of reverse triiodothyronine and mortality after acute myocardial infarction. The American journal of medicine 111, 699-703 (2001).

26. Jung, C. H. et al. Higher serum free thyroxine levels are associated with coronary artery disease. Endocrine journal 55, 819-826 (2008).

27. Auer, J., Berent, R., Weber, T., Lassnig, E. \& Eber, B. Thyroid function is associated with presence and severity of coronary atherosclerosis. Clinical cardiology 26, 569-573 (2003).

28. Ertas, F., Kaya, H. \& Soydinc, M. S. Low serum free triiodothyronine levels are associated with the presence and severity of coronary artery disease in the euthyroid patients: an observational study. Anadolu kardiyoloji dergisi: $A K D=$ the Anatolian journal of cardiology 12, 591-596, https://doi.org/10.5152/akd.2012.187 (2012).

29. Daswani, R., Jayaprakash, B., Shetty, R. \& Rau, N. R. Association of Thyroid Function with Severity of Coronary Artery Disease in Euthyroid Patients. Journal of clinical and diagnostic research: JCDR 9, Oc10-13, https://doi.org/10.7860/jcdr/2015/10908.6059 (2015).

30. Mayer, O. Jr, Cech, J., Rosolova, H., Pikner, R. \& Simon, J. [Association between free thyroxin concentration and degree of heart failure in patients with chronic heart insufficiency]. Casopis lekaru ceskych 144, 742-746 (2005).

31. Mayer, O. Jr et al. Even mild changes in free thyroxine could influence the degree of heart failure measured by its biological surrogates. Physiological research 57, 525-529 (2008)

32. Asvold, B. O., Bjoro, T., Platou, C. \& Vatten, L. J. Thyroid function and the risk of coronary heart disease: 12-year follow-up of the HUNT study in Norway. Clinical endocrinology 77, 911-917, https://doi.org/10.1111/j.1365-2265.2012.04477.x (2012).

33. Panicker, V. Genetics of thyroid function and disease. The Clinical biochemist. Reviews 32, 165-175 (2011).

34. Peeters, R. P. et al. Polymorphisms in thyroid hormone pathway genes are associated with plasma TSH and iodothyronine levels in healthy subjects. The. Journal of clinical endocrinology and metabolism 88, 2880-2888, https://doi.org/10.1210/jc.2002-021592 (2003).

35. van der Deure, W. M. et al. Polymorphisms in the brain-specific thyroid hormone transporter OATP1C1 are associated with fatigue and depression in hypothyroid patients. Clinical endocrinology 69, 804-811, https://doi.org/10.1111/j.1365-2265.2008.03267.x (2008).

36. Verloop, H., Dekkers, O. M., Peeters, R. P., Schoones, J. W. \& Smit, J. W. Genetics in endocrinology: genetic variation in deiodinases: a systematic review of potential clinical effects in humans. European journal of endocrinology 171, R123-135, https://doi. org/10.1530/eje-14-0302 (2014).

37. Bianco, A. C., Salvatore, D., Gereben, B., Berry, M. J. \& Larsen, P. R. Biochemistry, cellular and molecular biology, and physiological roles of the iodothyronine selenodeiodinases. Endocrine reviews 23, 38-89, https://doi.org/10.1210/edrv.23.1.0455 (2002).

38. Maia, A. L., Kim, B. W., Huang, S. A., Harney, J. W. \& Larsen, P. R. Type 2 iodothyronine deiodinase is the major source of plasma T3 in euthyroid humans. The. Journal of clinical investigation 115, 2524-2533, https://doi.org/10.1172/jci25083 (2005).

39. Maia, A. L., Goemann, I. M., Meyer, E. L. \& Wainer, S. M. Deiodinases: the balance of thyroid hormone: type 1 iodothyronine deiodinase in human physiology and disease. The Journal of endocrinology 209, 283-297, https://doi.org/10.1530/joe-10-0481 (2011).

40. Gereben, B. et al. Cellular and molecular basis of deiodinase-regulated thyroid hormone signaling. Endocrine reviews 29, 898-938, https://doi.org/10.1210/er.2008-0019 (2008).

41. Marsili, A., Zavacki, A. M., Harney, J. W. \& Larsen, P. R. Physiological role and regulation of iodothyronine deiodinases: a 2011 update. Journal of endocrinological investigation 34, 395-407, https://doi.org/10.3275/761510.1007/bf03347465 (2011).

42. Peeters, R. P. et al. A new polymorphism in the type II deiodinase gene is associated with circulating thyroid hormone parameters. American journal of physiology. Endocrinology and metabolism 289, E75-81, https://doi.org/10.1152/ajpendo.00571.2004 (2005).

43. Park, E. et al. Concurrent TSHR mutations and DIO2 T92A polymorphism result in abnormal thyroid hormone metabolism. Scientific Reports 8, 10090, https://doi.org/10.1038/s41598-018-28480-0 (2018).

44. Panicker, V. et al. Heritability of serum TSH, free T4 and free T3 concentrations: a study of a large UK twin cohort. Clinical endocrinology 68, 652-659, https://doi.org/10.1111/j.1365-2265.2007.03079.x (2008).

45. Porcu, E. et al. A meta-analysis of thyroid-related traits reveals novel loci and gender-specific differences in the regulation of thyroid function. PLoS genetics 9, e1003266, https://doi.org/10.1371/journal.pgen.1003266 (2013).

46. Brozaitiene, J. et al. Deiodinases, Organic Anion Transporter Polypeptide Polymorphisms, and Thyroid Hormones in Patients with Myocardial Infarction. GENETIC TESTING AND MOLECULAR. BIOMARKERS 22, 1-9 (2018).

47. Steg, P. G. et al. ESC Guidelines for the management of acute myocardial infarction in patients presenting with ST-segment elevation. European heart journal 33, 2569-2619, https://doi.org/10.1093/eurheartj/ehs215 (2012).

48. Thygesen, K. et al. Third universal definition of myocardial infarction. Global heart 7, 275-295, https://doi.org/10.1016/j. gheart.2012.08.001 (2012)

49. Amsterdam, E. A. et al. 2014 AHA/ACC Guideline for the Management of Patients with Non-ST-Elevation Acute Coronary Syndromes: a report of the American College of Cardiology/American Heart Association Task Force on Practice Guidelines. Journal of the American College of Cardiology 64, e139-e228, https://doi.org/10.1016/j.jacc.2014.09.017 (2014). 
50. Roffi, M. et al. 2015 ESC Guidelines for the Management of Acute Coronary Syndromes in Patients Presenting Without Persistent ST-segment Elevation. Revista espanola de cardiologia (English ed.) 68, 1125, https://doi.org/10.1016/j.rec.2015.10.009 (2015).

51. Burkauskas, J. et al. Gene-environment interactions connecting Low triiodothyronine syndrome and outcomes of cardiovascular disease (GETVASC): study protocol. Biological Psychiatry and Psychopharmacology 16, 66-73 (2014).

52. Panicker, V. et al. A common variation in deiodinase 1 gene DIO1 is associated with the relative levels of free thyroxine and triiodothyronine. The Journal of clinical endocrinology and metabolism 93, 3075-3081, https://doi.org/10.1210/jc.2008-0397 (2008).

53. Philibert, R. A. et al. The relationship of deiodinase 1 genotype and thyroid function to lifetime history of major depression in three independent populations. American journal of medical genetics. Part B, Neuropsychiatric genetics: the official publication of the International Society of Psychiatric Genetics 156b, 593-599, https://doi.org/10.1002/ajmg.b.31200 (2011).

54. Lesauskaite, V. et al. Matrix metalloproteinase-3 gene polymorphism and dilatative pathology of ascending thoracic aorta. Medicina (Kaunas, Lithuania) 44, 386-391 (2008).

55. Rodriguez, S., Gaunt, T. R. \& Day, I. N. Hardy-Weinberg equilibrium testing of biological ascertainment for Mendelian randomization studies. American journal of epidemiology 169, 505-514, https://doi.org/10.1093/aje/kwn359 (2009).

56. Pop, V. J. et al. Low maternal free thyroxine concentrations during early pregnancy are associated with impaired psychomotor development in infancy. Clinical endocrinology 50, 149-155 (1999).

57. Saravanan, P. et al. Psychological well-being in patients on 'adequate' doses of l-thyroxine: results of a large, controlled communitybased questionnaire study. Clinical endocrinology 57, 577-585 (2002).

58. Angelousi, A. G., Karageorgopoulos, D. E., Kapaskelis, A. M. \& Falagas, M. E. Association between thyroid function tests at baseline and the outcome of patients with sepsis or septic shock: a systematic review. European journal of endocrinology 164, 147-155, https://doi.org/10.1530/eje-10-0695 (2011).

59. Carrero, J. J. et al. Clinical and biochemical implications of low thyroid hormone levels (total and free forms) in euthyroid patients with chronic kidney disease. Journal of internal medicine 262, 690-701, https://doi.org/10.1111/j.1365-2796.2007.01865.x (2007).

60. Baue, A. E., Gunther, B., Hartl, W., Ackenheil, M. \& Heberer, G. Altered hormonal activity in severely ill patients after injury or sepsis. Archives of surgery (Chicago, Ill.: 1960) 119, 1125-1132 (1984).

61. Moura Neto, A. \& Zantut-Wittmann, D. E. Abnormalities of Thyroid Hormone Metabolism during Systemic Illness: The Low T3 Syndrome in Different Clinical Settings. International journal of endocrinology 2016, 2157583, https://doi. org/10.1155/2016/2157583 (2016).

62. Grabowski, M. et al. Admission B-type natriuretic peptide assessment improves early risk stratification by Killip classes and TIMI risk score in patients with acute ST elevation myocardial infarction treated with primary angioplasty. International journal of cardiology 115, 386-390, https://doi.org/10.1016/j.ijcard.2006.04.038 (2007).

63. Wang, W. Y. et al. Free triiodothyronine level indicates the degree of myocardial injury in patients with acute ST-elevation myocardial infarction. Chinese medical journal 126, 3926-3929 (2013).

64. Satar, S. et al. Prognostic value of thyroid hormone levels in acute myocardial infarction: just an epiphenomenon? The American heart hospital journal 3, 227-233 (2005).

65. Bai, M. F. et al. Effects of thyroid dysfunction on the severity of coronary artery lesions and its prognosis. Journal of cardiology 64, 496-500, https://doi.org/10.1016/j.jjcc.2014.03.009 (2014).

66. Franklyn, J. A., Gammage, M. D., Ramsden, D. B. \& Sheppard, M. C. Thyroid status in patients after acute myocardial infarction. Clinical science (London, England: 1979) 67, 585-590 (1984).

67. Pavlou, H. N., Kliridis, P. A., Panagiotopoulos, A. A., Goritsas, C. P. \& Vassilakos, P. J. Euthyroid sick syndrome in acute ischemic syndromes. Angiology 53, 699-707, https://doi.org/10.1177/000331970205300611 (2002).

68. Pimentel, R. C., Cardoso, G. P., Escosteguy, C. C. \& Abreu, L. M. Thyroid hormone profile in acute coronary syndromes. Arquivos brasileiros de cardiologia 87, 688-694 (2006).

69. Iltumur, K., Olmez, G., Ariturk, Z., Taskesen, T. \& Toprak, N. Clinical investigation: thyroid function test abnormalities in cardiac arrest associated with acute coronary syndrome. Critical care (London, England) 9, R416-424, https://doi.org/10.1186/cc3727 (2005).

70. Stevenson, H. P., Archbold, G. P., Johnston, P., Young, I. S. \& Sheridan, B. Misleading serum free thyroxine results during low molecular weight heparin treatment. Clinical chemistry 44, 1002-1007 (1998).

71. Koulouri, O., Moran, C., Halsall, D., Chatterjee, K. \& Gurnell, M. Pitfalls in the measurement and interpretation of thyroid function tests. Best practice \& research. Clinical endocrinology \& metabolism 27, 745-762, https://doi.org/10.1016/j.beem.2013.10.003 (2013).

72. Opasich, C. et al. Sick euthyroid syndrome in patients with moderate-to-severe chronic heart failure. European heart journal 17, 1860-1866 (1996)

73. Cerillo, A. G. et al. The low triiodothyronine syndrome: a strong predictor of low cardiac output and death in patients undergoing coronary artery bypass grafting. The Annals of thoracic surgery 97, 2089-2095, https://doi.org/10.1016/j.athoracsur.2014.01.049 (2014).

74. Sbrana, F. et al. Routine laboratory tests to risk-stratify patients with chronic coronary artery disease. Journal of cardiology 61, 132-137, https://doi.org/10.1016/j.jicc.2012.09.005 (2013).

75. Hayashi, T. et al. Subclinical hypothyroidism is an independent predictor of adverse cardiovascular outcomes in patients with acute decompensated heart failure. ESC heart failure 3, 168-176, https://doi.org/10.1002/ehf2.12084 (2016).

76. Ceremuzynski, L. et al. Low serum triiodothyronine in acute myocardial infarction indicates major heart injury. Kardiologia polska 60, 468-480; discussion 473-464 (2004)

77. Hak, A. E. et al. Subclinical hypothyroidism is an independent risk factor for atherosclerosis and myocardial infarction in elderly women: the Rotterdam Study. Annals of internal medicine 132, 270-278 (2000).

78. Kim, E. S. et al. Association between low serum free thyroxine concentrations and coronary artery calcification in healthy euthyroid subjects. Thyroid: official journal of the American Thyroid Association 22, 870-876, https://doi.org/10.1089/thy.2011.0366 (2012).

79. Iida, M. et al. Thyroid hormone within the normal range is associated with left ventricular mass in patients with hypertension. Journal of the American Society of Hypertension: JASH 6, 261-269, https://doi.org/10.1016/j.jash.2012.04.002 (2012).

80. Pfister, R. et al. The relationship and prognostic impact of low-T3 syndrome and NT-pro-BNP in cardiovascular patients. International journal of cardiology 144, 187-190, https://doi.org/10.1016/j.ijcard.2009.03.137 (2010).

81. Wang, F. et al. Relationship between thyroid function and ICU mortality: a prospective observation study. Critical care (London, England) 16, R11, https://doi.org/10.1186/cc11151 (2012).

82. Pfister, R., Tan, D., Thekkanal, J., Hellmich, M. \& Schneider, C. A. NT-pro-BNP is associated with long-term outcome in a heterogeneous sample of cardiac inpatients. European journal of internal medicine 18, 215-220, https://doi.org/10.1016/j. ejim.2006.11.007 (2007).

83. Passino, C. et al. Prognostic value of combined measurement of brain natriuretic peptide and triiodothyronine in heart failure. Journal of cardiac failure 15, 35-40, https://doi.org/10.1016/j.cardfail.2008.08.008 (2009).

84. van der Deure, W. M. et al. The effect of genetic variation in the type 1 deiodinase gene on the interindividual variation in serum thyroid hormone levels: an investigation in healthy Danish twins. Clinical endocrinology 70, 954-960, https://doi. org/10.1111/j.1365-2265.2008.03420.x (2009). 
85. Grarup, N. et al. Studies of the common DIO2 Thr92Ala polymorphism and metabolic phenotypes in 7342 Danish white subjects. The. Journal of clinical endocrinology and metabolism 92,363-366, https://doi.org/10.1210/jc.2006-1958 (2007).

86. Peeters, R. P., van der Deure, W. M. \& Visser, T. J. Genetic variation in thyroid hormone pathway genes; polymorphisms in the TSH receptor and the iodothyronine deiodinases. European journal of endocrinology 155, 655-662, https://doi.org/10.1530/eje.1.02279 (2006).

87. Biondi, B. Mechanisms in endocrinology: Heart failure and thyroid dysfunction. European journal of endocrinology 167, 609-618, https://doi.org/10.1530/eje-12-0627 (2012).

88. Olivares, E. L. et al. Thyroid function disturbance and type 3 iodothyronine deiodinase induction after myocardial infarction in rats a time course study. Endocrinology 148, 4786-4792, https://doi.org/10.1210/en.2007-0043 (2007).

89. Klein, I. \& Danzi, S. Thyroid disease and the heart. Circulation 116, 1725-1735, https://doi.org/10.1161/circulationaha.106.678326 (2007).

90. Klein, I. \& Ojamaa, K. Thyroid hormone and the cardiovascular system. The New England journal of medicine 344, 501-509, https://doi.org/10.1056/nejm200102153440707 (2001).

91. de Jong, F. J. et al. The association of polymorphisms in the type 1 and 2 deiodinase genes with circulating thyroid hormone parameters and atrophy of the medial temporal lobe. The Journal of clinical endocrinology and metabolism 92, 636-640, https://doi. org/10.1210/jc.2006-1331 (2007)

92. Peeters, R. P. et al. Serum 3,3',5'-triiodothyronine (rT3) and 3,5,3'-triiodothyronine/rT3 are prognostic markers in critically ill patients and are associated with postmortem tissue deiodinase activities. The. Journal of clinical endocrinology and metabolism 90 4559-4565, https://doi.org/10.1210/jc.2005-0535 (2005).

93. Li, Q., Chen, L., Chen, D., Wu, X. \& Chen, M. Influence of microRNA-related polymorphisms on clinical outcomes in coronary artery disease. American journal of translational research 7, 393-400 (2015).

94. Carreras-Torres, R. et al. Genetic risk score of NOS gene variants associated with myocardial infarction correlates with coronary incidence across Europe. PloS one 9, e96504, https://doi.org/10.1371/journal.pone.0096504 (2014).

95. Morgan, T. M. et al. Investigation of 95 variants identified in a genome-wide study for association with mortality after acute coronary syndrome. BMC medical genetics 12, 127, https://doi.org/10.1186/1471-2350-12-127 (2011).

96. Lee, I. T. et al. Value of Chromosome 9p21 Polymorphism for Prediction of Cardiovascular Mortality in Han Chinese Without Coronary Lesions: An Observational Study. Medicine 94, e1538, https://doi.org/10.1097/md.0000000000001538 (2015).

97. Munir, M. S. et al. The association of 9p21-3 locus with coronary atherosclerosis: a systematic review and meta-analysis. BMC medical genetics 15, 66, https://doi.org/10.1186/1471-2350-15-66 (2014).

98. Abdullah, K. G. et al. Four SNPS on chromosome 9p21 confer risk to premature, familial CAD and MI in an American Caucasian population (GeneQuest). Annals of human genetics 72, 654-657, https://doi.org/10.1111/j.1469-1809.2008.00454.x (2008).

99. Schunkert, H. et al. Repeated replication and a prospective meta-analysis of the association between chromosome $9 \mathrm{p} 21.3$ and coronary artery disease. Circulation 117, 1675-1684, https://doi.org/10.1161/circulationaha.107.730614 (2008).

100. Kathiresan, S. et al. Genome-wide association of early-onset myocardial infarction with single nucleotide polymorphisms and copy number variants. Nature genetics 41, 334-341, https://doi.org/10.1038/ng.327 (2009).

101. Szpakowicz, A. et al. The rs 12526453 Polymorphism in an Intron of the PHACTR1 Gene and Its Association with 5-Year Mortality of Patients with Myocardial Infarction. PloS one 10, e0129820, https://doi.org/10.1371/journal.pone.0129820 (2015).

102. Wouters, H. J. et al. No Effect of the Thr92Ala Polymorphism of Deiodinase-2 on Thyroid Hormone Parameters, Health-Related Quality of Life, and Cognitive Functioning in a Large Population-Based Cohort Study. Thyroid: official journal of the American Thyroid Association 27, 147-155, https://doi.org/10.1089/thy.2016.0199 (2017)

103. Cooper-Kazaz, R. et al. Preliminary evidence that a functional polymorphism in type 1 deiodinase is associated with enhanced potentiation of the antidepressant effect of sertraline by triiodothyronine. Journal of affective disorders 116, 113-116, https://doi. org/10.1016/j.jad.2008.10.019 (2009).

104. Palmer, B. R. et al. Angiotensin-converting enzyme gene polymorphism interacts with left ventricular ejection fraction and brain natriuretic peptide levels to predict mortality after myocardial infarction. Journal of the American College of Cardiology 41, 729-736 (2003).

105. Kitsios, G. \& Zintzaras, E. ACE (I/D) polymorphism and response to treatment in coronary artery disease: a comprehensive database and meta-analysis involving study quality evaluation. BMC medical genetics 10, 50, https://doi.org/10.1186/1471-2350$10-50$ (2009).

\section{Acknowledgements}

We are grateful to Professor Robertas Bunevicius, MD, PhD (1958-2014) who guided the initial preparation of the study protocol. This research was funded by the European Social Fund under the Global Grant measure, Grant VP1-3.1-ŠMM-07-K-02-060.

\section{Author contributions}

N.K. contributed to the study and wrote the first manuscript. D.S. served as an expert in genetics, O.G. served as an expert in cardiology and N.M. served as an expert in endocrinology and all authors provided substantive feedback on the analysis, and made major contributions to the writing of the manuscript. J.B. and V.Z. contributed to the improvement of the analysis and of the written manuscript. JBr made leading contribution to conception of the study. All authors read and approved the final manuscript.

\section{Competing interests}

The authors declare that they have no competing interests. In the past several years Dr. Julius Burkauskas has been serving as a consultant at Cogstate, Ltd.

\section{Additional information}

Supplementary information is available for this paper at https://doi.org/10.1038/s41598-020-66006-9.

Correspondence and requests for materials should be addressed to J.B.

Reprints and permissions information is available at www.nature.com/reprints.

Publisher's note Springer Nature remains neutral with regard to jurisdictional claims in published maps and institutional affiliations. 
(c) (i) Open Access This article is licensed under a Creative Commons Attribution 4.0 International License, which permits use, sharing, adaptation, distribution and reproduction in any medium or format, as long as you give appropriate credit to the original author(s) and the source, provide a link to the Creative Commons license, and indicate if changes were made. The images or other third party material in this article are included in the article's Creative Commons license, unless indicated otherwise in a credit line to the material. If material is not included in the article's Creative Commons license and your intended use is not permitted by statutory regulation or exceeds the permitted use, you will need to obtain permission directly from the copyright holder. To view a copy of this license, visit http://creativecommons.org/licenses/by/4.0/.

(C) The Author(s) 2020 